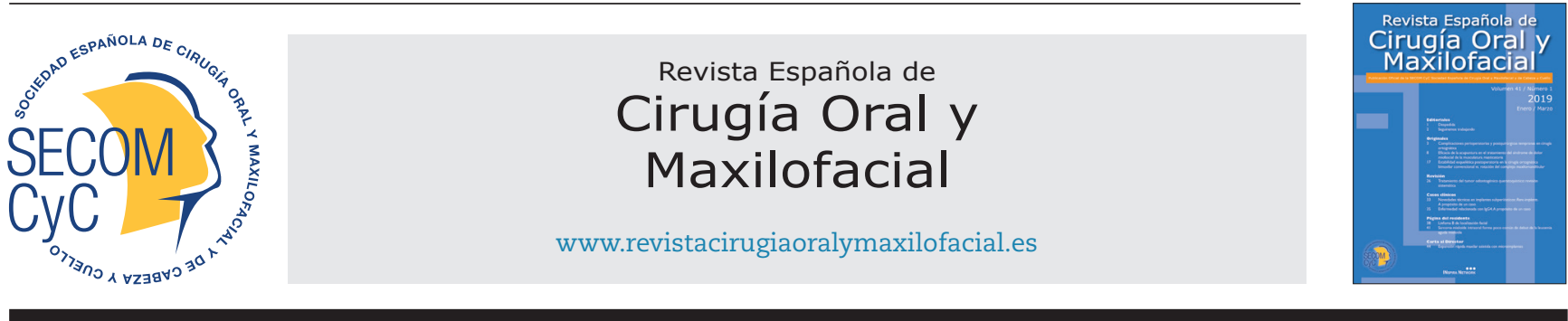

\title{
Original
}

\section{Eficacia de la acupuntura en el tratamiento del síndrome de dolor miofascial de la musculatura masticatoria}

\author{
Gui-Youn Cho-Lee , Hoon Cho-Jung ${ }^{b}$, Sergio Castrejón-Castrejónc, Mario Fernando Muñoz- \\ Guerrad $^{d}$,Francisco José Rodríguez-Campo ${ }^{d}$, Francisco Javier Díaz-González ${ }^{d}$ y Luis Naval-Gías ${ }^{d}$
}

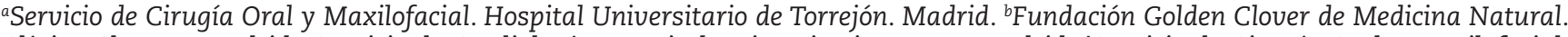
Clínica Cho Lee. Madrid. 'Servicio de Cardiología. Hospital Universitario La Paz. Madrid. 'Servicio de Cirugía Oral y Maxilofacial. Hospital Universitario de La Princesa. Madrid, España

\section{INFORMACIÓN DEL ARTÍCULO}

Historia del artículo:

Recibido el 20 de abril de 2018

Aceptado el 25 de abril de 2018

Palabras clave:

Acupuntura, síndrome de dolor miofascial, musculatura masticatoria.

\section{R E S U M E N}

Objetivos: El síndrome de dolor miofascial (SDM) de la musculatura masticatoria (MM) constituye la patología más frecuente dentro de los trastornos temporomandibulares (TTM). En cuanto al tratamiento del SDM de la MM, no existen protocolos estandarizados. Hay evidencia de que la acupuntura es eficaz en el tratamiento del dolor miofascial. El objetivo del presente estudio es analizar la eficacia de la acupuntura en el tratamiento del SDM de la MM en términos de reducción de la intensidad del dolor y la duración de la reducción del dolor en el tiempo.

Material y métodos: Se realizó un estudio observacional prospectivo en 30 pacientes diagnosticados de SDM de la MM que fueron tratados mediante 15 sesiones de acupuntura. Las variables del estudio fueron: 1) Porcentaje de pacientes que alcanzan una respuesta clínica relevante a lo largo del seguimiento (disminución del dolor de, al menos, un $50 \%$ en la escala visual analógica con respecto al inicial o bien una reducción total del dolor a $<30 \mathrm{~mm}$ en la misma escala). 2) Reducción del dolor muscular orofacial después del tratamiento, expresado en milímetros (mm), dentro de la escala visual analógica (EVA). 3) Máxima apertura oral (MAO) expresada en mm. 4) Estabilidad del efecto terapéutico. Las variables fueron evaluadas antes del tratamiento, a los 3, 6, 9 y 12 meses.

Resultados: La mediana de edad fue de 42 años (33-53). Con respecto al sexo, 28 de los 30 pacientes (93\%) fueron mujeres. El seguimiento fue completo para los 30 pacientes. Al final del seguimiento, 20 de los 30 pacientes (67\%, IC 95\% 49-81) mantuvieron una reducción del dolor a la categoría leve (EVA $<30 \%$ ) o bien una reducción $>50 \%$ del basal. A lo largo del estudio, el porcentaje de pacientes que alcanzó el criterio de respuesta clínica relevante preestablecido se mantuvo estable (67-80 \%), no variando significativamente a lo largo del tiempo. Conclusiones: Los resultados del estudio demuestran que la acupuntura es eficaz en el control del dolor del SDM de la MM. Los efectos terapéuticos de la acupuntura son de inicio temprano ( $<3$ meses), estables y se mantienen hasta el final del seguimiento de un año.

\footnotetext{
*Autor para correspondencia Correo electrónico: gui-youncho@hotmail.com; choguiyoun@gmail.com (Gui-Youn Cho Lee). https://doi.org/10.20986/recom.2019.1027/2019
}

1130-0558/@ 2019 SECOM. Publicado por Inspira Network. Este es un artículo Open Access bajo la licencia CC BY-NC-ND (http:// creativecommons.org/licenses/by-nc-nd/4.0/). 
Keywords:

Acupuncture, myofascial pain syndrome, masticatory muscles.

\section{A B S T R A C T}

Objectives: Myofascial Pain Syndrome (MPS) of the Masticatory Muscles (MM) is one of the most prevalent diseases included in the Temporomandibular Joint Dysfunction Syndrome. Regarding its treatment, there are not standarized protocols. There is some evidence that acupuncture is effective in MPS of the MM treatment. The objective of the present study is to analyze the efficacy of acupuncture for the treatment of MPS of the MM, in terms of pain intensity reduction and duration of the pain reduction along time.

Patients and methods: This is an observational prospective study. Thirty patients diagnosed of MPS of the MM were treated with 15 sessions of acupuncture. The variables of the study included were: 1) Percentage of patients that achieved a relevant clinical response (pain reduction of at least $50 \%$ from the initial value, in a visual analogue scale, or a visual analogue scale pain value less than 30 milimeters). 2) Pain reduction after treatment, measured in milimeters in a visual analogue scale (VAS). 3) Maximal mouth opening measured in milimeters. 4) Stability of the pain reduction. The variables were evaluated before treatment and 3, 6, 9 and 12 months after.

Results: The median of age was 42 years (33-53). Twenty eight patients were female (93\%). The follow up period lasted one year and all the patients completed it. At the end of the follow up period, 20 of the 30 patients (67\%, IC $95 \%$ 49-81) maintained a pain reduction in a mild category (VAS $<30 \%$ ) or a total reduction of $>50 \%$ from de initial pain value. Along the follow-up period, the percentage of patients that achieved a relevant clinical response maintained stable (67-80\%).

Conclusions: The results of the study show that acupuncture is effective in the treatment of MPS of the MM. Treatment effects appear early ( $<3$ months) and maintain stable within the first year.

\section{Introducción}

El SDM de la MM constituye la patología más frecuente dentro de los TTM. Es una enfermedad que comienza por una alteración muscular funcional afectando de modo primario a los músculos masticadores. Su manifestación clínica más importante es el dolor orofacial de origen muscular, especialmente a la palpación, afectando significativamente a funciones básicas para los pacientes tales como comer, hablar o dormir y, por tanto, puede tener como resultado un deterioro en la calidad de vida. En ocasiones clínicamente asocia dolor a nivel de la articulación temporomandibular, sin poder delimitar bien un origen u otro del dolor ${ }^{1}$.

Se estima que el 50-75\% de la población presenta signos y síntomas de SDM de la MM alguna vez a lo largo de la vida ${ }^{2}$. Es una patología frecuente en mujeres (algunos estudios ${ }^{3-5}$ estiman un porcentaje de hasta el $90 \%$ de sexo femenino) de mediana edad, entre los 20 y los 60 años ${ }^{4,5}$.

La etiopatogenia del SDM es multifactorial y compleja. Desde el punto de vista clínico, el sustrato anatómico del dolor muscular es el punto gatillo (PG), cuya definición la establecieron los autores Travell y Simons ${ }^{6}$. Los PG son focos hiperirritables dentro de una banda tensa de tejido muscular, localizada en la fibra muscular propiamente dicha o en su fascia asociada. El PG es doloroso a la presión y produce un dolor referido característico, así como fenómenos autónomos y motores.

En la actualidad, se está considerando la aplicación de la acupuntura en la práctica médica occidental para diversas patologías, especialmente en aquellas para las cuales las técnicas de la medicina moderna occidental presentan una efi- cacia limitada 7 . El tratamiento del dolor crónico es una de sus indicaciones principales ${ }^{8,9}$.

Numerosos estudios evidencian que la acupuntura es eficaz en el tratamiento del dolor miofascial ${ }^{8-33}$. Los mecanismos de acción de la acupuntura en el control del dolor son varios e implican al sistema nervioso central y periférico, sistemas hormonal e inmune. Estudios realizados en humanos y en animales con tomografía de emisión de positrones (TEP) y resonancia magnética demuestran que el estímulo en los puntos de acupuntura actúa como estímulo del sistema nervioso central, que a su vez activaría la vía antinociceptiva descendente, resultando en la liberación de opioides endógenos que desactivan las vías ascendentes nociceptivas que rigen el dolor ${ }^{34-37}$.

Un aspecto fundamental de la acupuntura es la selección de los puntos que constituyen un determinado tratamiento. Hay puntos locales (cercanos al área afecta de la patología) y distales (alejados del área afecta). Los puntos locales producen una respuesta analgésica más intensa pero más breve, por inhibición directa de la médula espinal; mientras que los puntos distales obtienen una respuesta menos intensa pero más prolongada y generalizada, debido a una respuesta general corporal vía activación hipotalámica ${ }^{34-37}$.

A pesar de que son numerosos los estudios sobre la eficacia de la acupuntura en el tratamiento del dolor, aquellos que analizan su eficacia en el tratamiento del SDM de la MM son escasos.

El objetivo principal del presente estudio es analizar la eficacia de la acupuntura en el tratamiento del SDM de la MM en términos de reducción de la intensidad del dolor, así como la duración de la reducción del dolor en el tiempo. 


\section{Material y métodos}

El presente estudio se trató de un estudio observacional prospectivo, realizado entre enero de 2010 y diciembre de 2013, en el Servicio de Cirugía Oral y Maxilofacial del Hospital Universitario de La Princesa de Madrid, España. Se incluyeron 30 pacientes de edades comprendidas entre 18 y 65 años, con diagnóstico clínico de SDM de la MM, sin componente clínico articular, con una duración de los síntomas de, al menos, seis meses, que no hubieran recibido tratamiento previo.

Los pacientes realizaron el tratamiento de acupuntura de forma ambulatoria, hasta completar 15 sesiones (aproximadamente, 2-3 sesiones semanales durante 4-6 semanas). Cada sesión tuvo una duración de 30 minutos. La estimulación de los puntos de acupuntura (PA) se realizó mediante la inserción de agujas en los mismos. El material que las compone fue acero inoxidable y presentaron una longitud de $40 \mathrm{~mm}$ y un grosor de 0,30 mm (Dong Bang ${ }^{\circledR}$ Acupuncture Inc., Corea del Sur) (Figura 1). Se insertaron perpendicularmente a la superficie de la piel previamente desinfectada y a una profundidad de 10 a 30 mm, según la constitución corporal del paciente; en posición de decúbito supino. Tras la inserción de las agujas, se procedió a realizar movimientos de rotación bidireccionales de la aguja con el fin de estimular el punto, provocando una sensación de irradiación (Deqi). Las agujas se mantuvieron insertadas durante 25 minutos.

Los puntos de acupuntura empleados fueron seleccionados por el acupuntor responsable del tratamiento. Constaron de dos puntos locales y siete distales. Los puntos seleccionados fueron los siguientes (número del punto, nombre del meridiano, nombre del punto, localización):

- 2 Estómago (Ha-Gwan) (Figura 2). Punto situado delante del pabellón auricular, debajo del borde inferior del arco cigomático, a una distancia por delante del trago. Punto local.

- 17 Triple Recalentador (Ye-Pung). Punto situado detrás del lóbulo de la oreja, en el hueco que existe delante del borde anterior de la mastoides. Punto local.

- 36 Estómago (Jog-Sam-Li). Punto localizado a tres distancias por debajo de la punta de la rótula, entre el músculo tibial anterior y el extensor común de los dedos. Punto distal.

- 41 Estómago (Hae-Gye). Punto situado por debajo del borde inferior de la tibia, a media distancia entre los dos maleolos, por dentro del tendón del músculo extensor común de los dedos. Punto distal.

- 4 Intestino Grueso (Hab-Gog) (Figura 3). Punto situado en el ángulo formado por los extremos proximales del $1 .^{\circ} \mathrm{y}$ 2. ${ }^{\circ}$ metacarpianos. Punto distal.

- 11 Intestino Grueso (Gog-Ji). Punto situado en el codo. Se localiza flexionando al máximo el codo, en la extremidad externa del pliegue, en un hueco. Punto distal.

- 3 Hígado (Tae-Chung). Punto situado en el extremo proximal del espacio interóseo formado por el $1 .^{\circ}$ y $2 .^{\circ}$ metatarsianos, hacia el primer metatarsiano. Punto distal.

- 34 Vesícula Biliar (Yang-Leung-Cheon). Punto localizado delante y debajo de la cabeza del peroné, a 2 distancias de la interlínea articular de la rodilla.

- 39 Vesícula Biliar (Hyeon-Jong). Punto situado a 3 distancias por encima del maleolo externo, sobre el borde anterior del peroné.

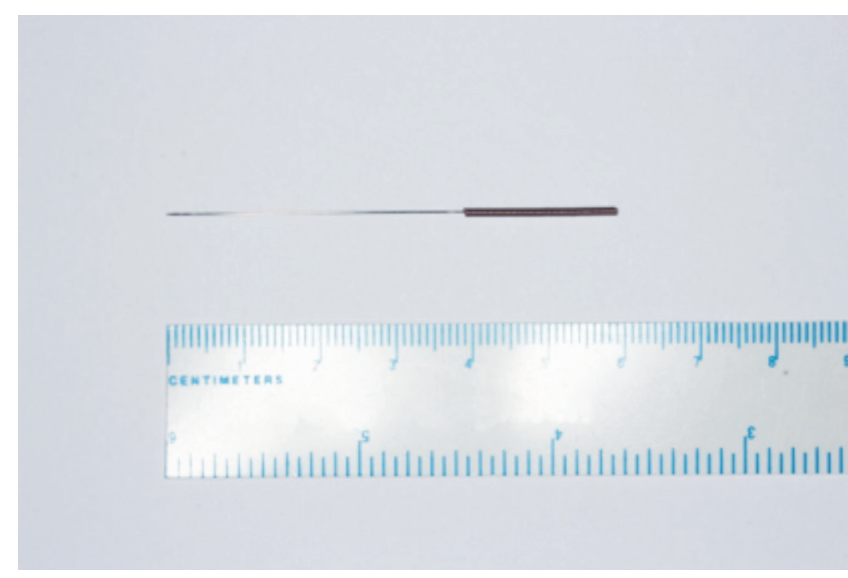

Figura 1. Aguja de acupuntura.

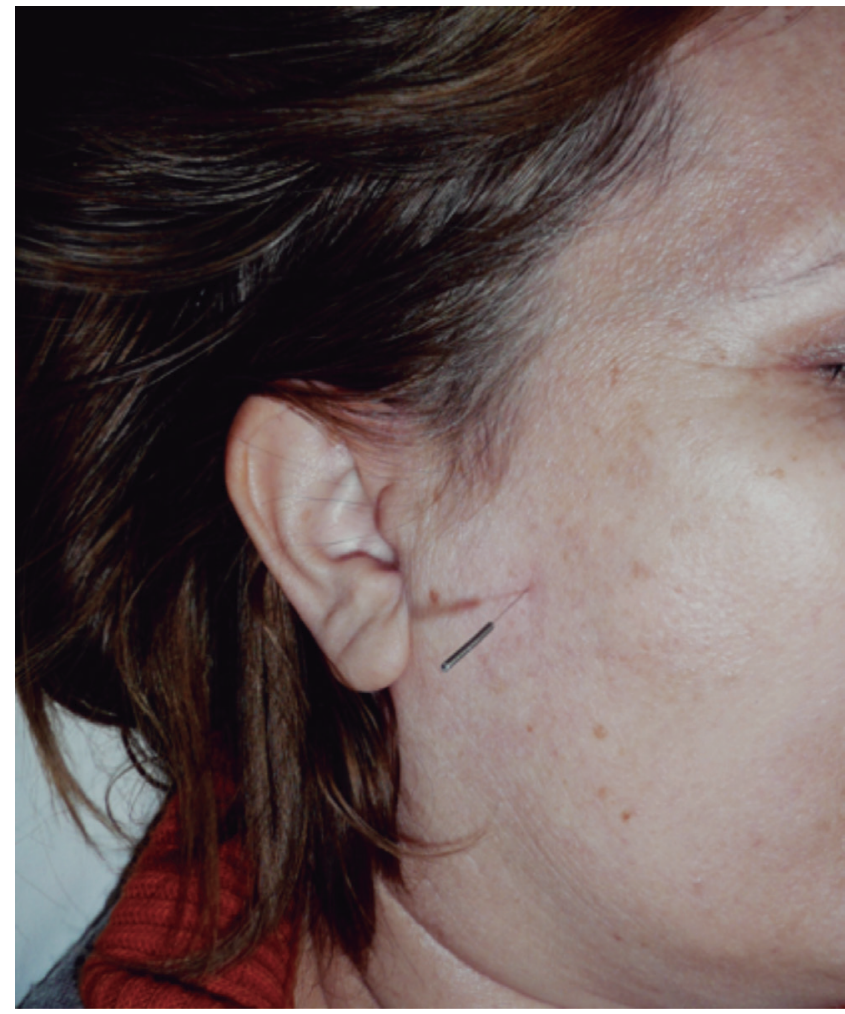

Figura 2. Punto 2 Estómago (Ha-Gwan). Punto situado delante del pabellón auricular, debajo del borde inferior del arco cigomático, a una distancia (*) por delante del trago. Punto local.

La variable principal del estudio fue el porcentaje de pacientes que alcanzan una respuesta clínica relevante a lo largo del seguimiento y que la mantiene al final del mismo. Definimos respuesta clínica relevante a la disminución del dolor de, al menos, un 50 \% en la EVA con respecto al inicial o bien una reducción total del dolor a $<30 \mathrm{~mm}$ en la misma escala ${ }^{38}$. 


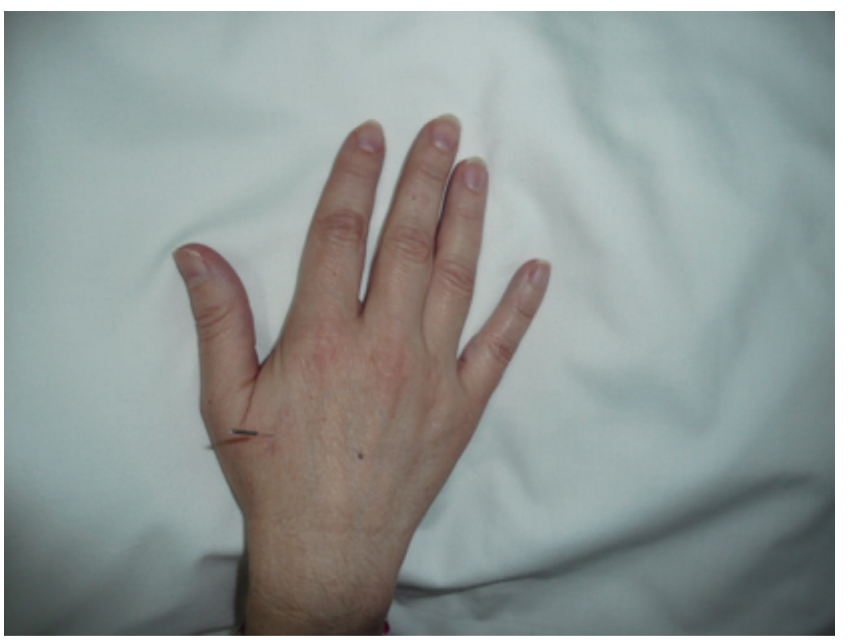

Figura 3. Punto 4 Intestino Grueso (Hab-Gog). Punto situado en el ángulo formado por los extremos proximales del $1 .^{\circ}$ y $2 .^{\circ}$ metacarpianos. Punto distal.

Las variables secundarias fueron:

- Reducción del dolor muscular orofacial después del tratamiento, expresado en milímetros ( $\mathrm{mm}$ ), dentro de una EVA que comprende valores de 0 a $100 \mathrm{~mm}$.

- Porcentaje de pacientes que entran en la categoría de dolor grave (EVA $>54 \mathrm{~mm}$ ) antes y después del tratamiento.

- Perspectiva funcional del paciente o Grado de disfunción percibida: EVA de 0 a $100 \mathrm{~mm}(0=$ ninguna limitación para comer, hablar o dormir; 100 = imposibilidad para comer, hablar o dormir) $)^{6}$.

- Máxima apertura oral (MAO) expresada en mm.

- Efectos colaterales y adversos del tratamiento.

- Estabilidad del efecto terapéutico.

\section{Métodos estadísticos}

- Las variables cuantitativas tanto continuas como discretas se presentan tanto en las tablas como en el texto y en las figuras en forma de mediana y rango intercuartílico (M, RIC) o bien en forma de media e intervalo de confianza del $95 \%$ de la media (m, IC $95 \%$ m). Las variables cualitativas se expresaron en forma de número total, porcentaje y su correspondiente intervalo de confianza ( $\mathrm{n}, \mathrm{p}$, IC $95 \%$ ).

- El análisis de medidas repetidas intragrupo para evaluar la estabilidad del efecto terapéutico se llevó a cabo con el test $\mathrm{Q}$ de Cochrane en caso de variables cualitativas y mediante la aplicación del modelo lineal general para variables cuantitativas.

- Los análisis de supervivencia aplicados a la aparición del criterio de respuesta clínica relevante a lo largo del seguimiento, se llevó a cabo mediante curvas de KaplanMeier y la significación estadística se evaluó mediante el test log-rank.

- Todos los cálculos se realizaron mediante el programa SPSS Statistics 15.0.

\section{Resultados}

En la Tabla I se exponen los valores de las variables al inicio del estudio.

En la Tabla II se exponen los resultados finales del estudio.

\section{Resultados con respecto al objetivo principal}

\section{Respuesta clínicamente relevante del dolor}

Al final del seguimiento, 20 pacientes (67\%, IC $95 \%$ 49-81) mantuvieron una reducción del dolor a la categoría leve (EVA $<30 \%$ ) o bien una reducción > 50 \% del basal. Es decir, alcanzaron el objetivo considerado respuesta clínica relevante.

A lo largo del estudio el porcentaje de pacientes que alcanza el criterio de respuesta clínica relevante preestablecido se mantiene estable, no variando significativamente a lo largo del tiempo, como queda expresado en la Tabla III mediante el valor de significación del estadístico $\mathrm{Q}$.

Tabla I. Características de los pacientes al inicio del estudio

\begin{tabular}{|c|c|}
\hline Variable & Valor \\
\hline Número & 30 \\
\hline $\begin{array}{l}\text { Sexo mujer } \\
\mathrm{n}(\%)\end{array}$ & $28(93 \%)$ \\
\hline $\begin{array}{l}\text { Edad } \\
\text { Mediana [RIQ] } \\
\text { Media (IC } 95 \% \text { ) }\end{array}$ & $\begin{array}{l}42[33-53] \\
42(37-47)\end{array}$ \\
\hline $\begin{array}{l}\text { Dolor EVA } \\
\text { Mediana [RIQ] } \\
\text { Media (IC } 95 \% \text { ) } \\
\text { - Moderado } \\
\text { - Severo: }\end{array}$ & $\begin{array}{c}70[60-80] \\
68(63-74) \\
18(60 \%) \\
12(40 \%)\end{array}$ \\
\hline Dolor grave $(E V A>54)^{\dagger}$ & $25(83 \%)$ \\
\hline $\begin{array}{l}\text { Disfunción EVA } \\
\text { Mediana [RIQ] } \\
\text { Media (IC } 95 \% \text { ) }\end{array}$ & $\begin{array}{l}70[56-80] \\
64(54-73)\end{array}$ \\
\hline Apertura $(\mathrm{mm})^{\ddagger}$ & $\begin{array}{l}38[35-45] \\
38(35-42)\end{array}$ \\
\hline
\end{tabular}

${ }^{\dagger}$ Número y \% ${ }^{\ddagger}$ Mediana [RIQ] y media (IC $95 \%$ de la media).

Tabla II. Resultados al final del estudio (1 año)

\begin{tabular}{|c|c|}
\hline Variable & Valor \\
\hline Dolor EVA ${ }^{\ddagger}$ & $\begin{array}{l}30[10-40] \\
29(20-38)\end{array}$ \\
\hline $\begin{array}{l}\text { - No dolor/levet: } \\
\text { - Moderado/severot: }\end{array}$ & $\begin{array}{l}21(70 \%) \\
9(30 \%)\end{array}$ \\
\hline Dolor grave $(\text { EVA }>54)^{\dagger}$ & $4(13 \%)$ \\
\hline Disfunción EVA ${ }^{\ddagger}$ & $\begin{array}{c}18[2-35] \\
25(16-35)\end{array}$ \\
\hline Apertura $(\mathrm{mm})^{\ddagger}$ & $\begin{array}{l}44[37-48] \\
42(39-45)\end{array}$ \\
\hline
\end{tabular}

${ }^{\dagger}$ Número y \% ₹Mediana [RIQ] y media (IC $95 \%$ de la media). 


\section{Dolor medido en la EVA}

Se evaluaron las diferencias cuantitativas totales de la intensidad del dolor al final del estudio. Se observa a lo largo del seguimiento una reducción de la intensidad del dolor (Tabla IV).

El descenso promedio de la puntuación (intensidad) de dolor entre el inicio y el final del seguimiento es de 40 [30-50] puntos (comparación antes/después, $\mathrm{p}<0,001$ ). El descenso de la puntuación del dolor en la EVA (intra-grupo) es estadísticamente significativo.

\section{Dolor grave $($ EVA $>54)$}

La proporción de pacientes que tienen dolor grave es significativamente más baja al final del seguimiento que en situación basal. La reducción de la proporción de pacientes con dolor grave a lo largo del seguimiento es estadísticamente significativa: valor basal (83 \%) versus final (13\%), $\mathrm{p}<0,001$.

Tabla III. Porcentaje de pacientes que alcanzó

el criterio de remisión clínica

\begin{tabular}{|c|c|}
\hline Seguimiento & Valor \\
\hline $\begin{array}{l}\text { Remisión dolor } 3 \text { meses } \\
\mathrm{n}(\%) \\
\mathrm{IC} 95 \%\end{array}$ & $\begin{array}{c}23(77 \%) \\
(59-88)\end{array}$ \\
\hline $\begin{array}{l}\text { Remisión dolor } 6 \text { meses } \\
\mathrm{n}(\%) \\
\text { IC } 95 \%\end{array}$ & $\begin{array}{c}24(80 \%) \\
(63-90)\end{array}$ \\
\hline $\begin{array}{l}\text { Remisión dolor } 9 \text { meses } \\
\mathrm{n}(\%) \\
\text { IC } 95 \%\end{array}$ & $\begin{array}{c}22(73 \%) \\
(56-86)\end{array}$ \\
\hline $\begin{array}{l}\text { Remisión dolor } 1 \text { año } \\
\text { n (\%) } \\
\text { IC } 95 \%\end{array}$ & $\begin{array}{c}20(67 \%) \\
(49-81)\end{array}$ \\
\hline $\mathrm{p}^{*}$ & 0,336 \\
\hline
\end{tabular}

Tabla IV. Evolución del dolor (según la EVA) entre el inicio y el fin del estudio

\begin{tabular}{lc}
\multicolumn{1}{c}{ Variable } & Valor \\
\hline Dolor EVA basal & $70[60-80]$ \\
Mediana [RIQ] & $68(63-74)$ \\
Media (IC 95 \%) & \\
Dolor EVA final & $30[10-40]$ \\
Mediana [RIQ] & $29(20-38)$ \\
Media (IC 95 \%) & \\
Reducción EVA al año & $40[30-50]$ \\
Mediana [RIQ] & $39(31-47)$ \\
Media (IC 95 \%) & \\
Dolor grave (EVA 54) & $25(83 \%)$ \\
- Inicio: & $4(13 \%)$ \\
- 1 año: &
\end{tabular}

A lo largo del seguimiento, 21 de los 25 pacientes que presentaba dolor grave al inicio del estudio presentó una respuesta clínica relevante de reducción del mismo: 84 \%, IC 95 \% 65-94 \%.

\section{Resultados con respecto a los objetivos secundarios}

MAO

Como se aprecia comparando la apertura final con la apertura inicial (Tabla V), hubo un ligero aumento estadísticamente significativo. El aumento promedio (mediana y rango intercuartílico) fue de 4 [1-6].

El comportamiento de la apertura oral también sugiere que el efecto de la acupuntura sobre esta variable. Así, se aprecia un aumento inicial de apertura cuyos valores se mantienen luego estables.

Perspectiva funcional o grado de disfunción percibida medido en EVA

Hubo una mejoría del grado de disfunción percibida a lo largo del seguimiento, de manera que la disfunción percibida final presenta diferencias estadísticamente significativas con respecto a la inicial.

La magnitud de la reducción del grado disfunción percibida a lo largo del seguimiento (diferencia disfunción percibida basal - disfunción percibida final) es en promedio (mediana) 50 puntos (RIC 10-63 puntos).

\section{Estabilidad del efecto terapéutico}

Es importante clínicamente evaluar la estabilidad del efecto terapéutico de la acupuntura porque el tratamiento fue aplicado solamente durante cuatro a seis semanas al principio del estudio. El nivel de significación estadística se ha calculado mediante análisis de medidas repetidas intra-grupo.

En la Figura 4 se aprecia que no existen diferencias significativas en la puntuación EVA del dolor a partir del tercer mes, manteniéndose estable el efecto del tratamiento y la mejoría inicial.

\section{Tabla V. Evolución de la apertura oral}

\begin{tabular}{lc}
\multicolumn{1}{c}{ Variable } & Valor \\
\hline Apertura (mm) & \\
Mediana [RIQ] & $38[35-45]$ \\
Media (IC 95 \%) & $38(35-42)$ \\
Apertura final (mm) & \\
Mediana [RIQ] & $44[37-48]$ \\
Media (IC 95 \%) & $42(39-45)$ \\
Diferencia apertura (mm) & \\
Mediana [RIQ] & $4[1-6]$ \\
Media (IC 95 \%) & $4(2-6)$ \\
Diferencia apertura: & \\
- > 5 mm: & $11(37 \%)$ \\
- Reducción: & $4(13 \%)$ \\
\hline
\end{tabular}


En la Figura 5 se presenta la estabilidad de la proporción de pacientes con remisión clínica a lo largo del seguimiento:

\section{Efectos adversos del tratamiento}

Ningún paciente refirió efectos adversos o intolerancia al tratamiento.

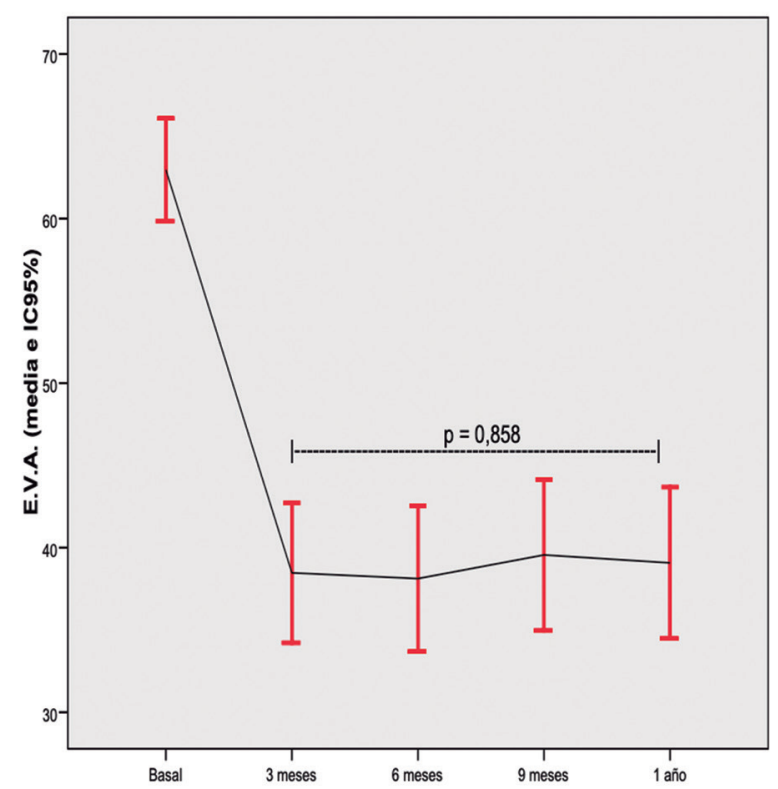

Figura 4. Estabilidad del efecto de la acupuntura (I): dolor medido en la EVA.

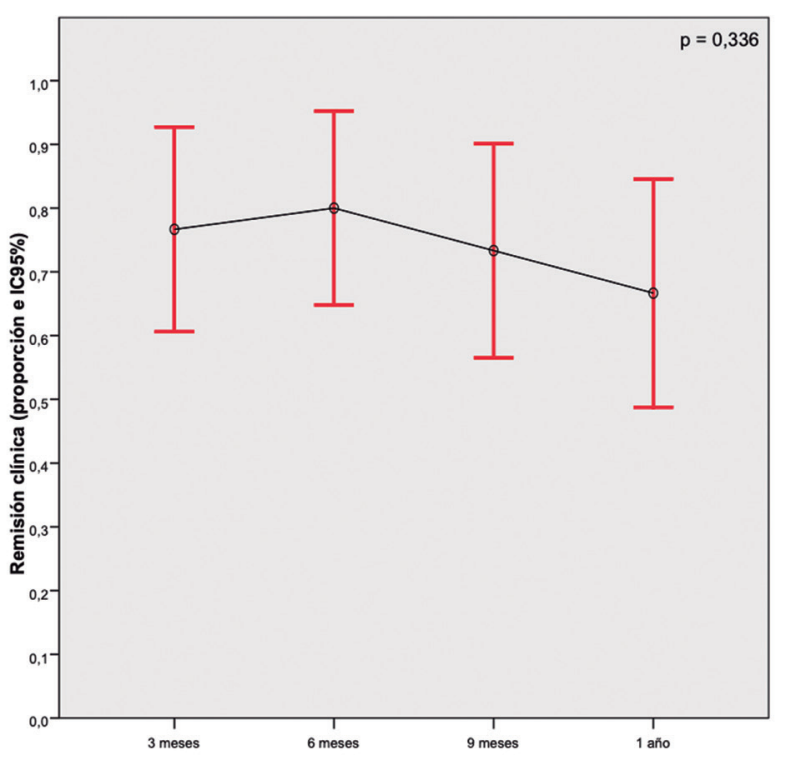

Figura 5. Estabilidad del efecto de la acupuntura (II): proporción de remisión clínica.

\section{Discusión}

A pesar de que son numerosos los estudios sobre la eficacia de la acupuntura en el tratamiento del dolor, aquellos que analizan su eficacia en el tratamiento del SDM de la MM son escasos.

Dentro de los estudios previos realizados en pacientes con SDM de la MM, aquellos que aportan una metodología más rigurosa son los ensayos clínicos aleatorizados. Dentro de estos, existen dos grupos: 1) Estudios que compararon la eficacia el tratamiento de la acupuntura con la eficacia de las medidas conservadoras y la feruloterapia. ${ }^{39-47}$ Todos estos estudios concluyeron que la acupuntura es eficaz en el tratamiento del dolor miofascial sin haber diferencias significativas con respecto a la feruloterapia. 2) Estudios que compararon la eficacia de la acupuntura y la acupuntura placebo ${ }^{2,38,48-52}$. La Touche y cols. ${ }^{53}$ y Jung y cols. ${ }^{54}$ realizaron una revisión sistemática y metanálisis de este tipo de estudios.

Las dos revisiones sistemáticas de La Touche y cols. ${ }^{53}$ y Jung y cols. ${ }^{54}$ coinciden en las limitaciones de los estudios anteriores: su pequeño tamaño muestral y falta de seguimiento a largo plazo. Salvo el estudio de Katsoulis y cols..$^{38}$ que realizó un seguimiento hasta los tres meses, el resto de los estudios evaluó solo los efectos inmediatos de los tratamientos. Otro aspecto es que algunos de los estudios previos emplean un solo punto de acupuntura, lo cual no es lo habitual en este tipo de tratamientos, en los que se suelen emplear combinaciones de puntos. Los autores ponen de manifiesto la necesidad de realizar estudios con un mayor seguimiento y de validar qué puntos o combinaciones de puntos deben tratarse. La Touche y cols. ${ }^{53}$ recomiendan el tratamiento de los puntos 2 Estómago (Ha-Gwan), como punto local, y 4 Intestino grueso (Hab-Gog) como punto distal.

Con respecto al número y duración de las sesiones, se establecieron un total de 15 sesiones con una frecuencia de 2-3 semanales, de 30 minutos de duración. La duración de las sesiones fue similar a la de los estudios de Goddard y cols. ${ }^{48}$, Schmid-Schwap y cols. ${ }^{50}$ y Smith y cols. ${ }^{2}$. Con respecto al número y frecuencia de las sesiones, observamos una gran variabilidad según los diferentes estudios: desde una sola sesión hasta 18 sesiones.

En nuestro estudio se emplearon los 9 puntos de acupuntura indicados en el apartado de Material y Métodos, escogidos según el criterio acupuntor. Dentro de los 9 puntos (2 locales y 7 distales), se incluyeron los dos puntos sugeridos en el metanálisis de La Touche y cols. ${ }^{53}: 2$ Estómago (Ha-Gwan), como punto local; y 4 Intestino grueso (Hab-Gog) como punto distal. Estos 9 puntos fueron tratados en todos los pacientes con el fin de estandarizar el tratamiento de acupuntura, lo cual no es la práctica habitual en este tipo de tratamientos, en los que la selección de puntos se realiza de manera individualizada ${ }^{38}$. $\mathrm{Al}$ respecto, List y cols. ${ }^{44}$ indicaron en su estudio que la combinación de puntos empleada se estandarizó lo máximo posible pero en ciertos casos, según las características particulares de cada uno de los pacientes, se emplearon otros puntos.

El predominio del sexo femenino en esta patología es ya conocida y algunos autores la describen con un porcentaje mayor al $80 \%{ }^{55}$. El SDM de la MM es una patología más frecuente en mujeres (hasta un $90 \%$ ) y de mediana edad, entre 
los 20 y los 50 años ${ }^{3-5}$. En el presente estudio, 28 de los 30 (93\%) pacientes fueron mujeres y la edad mediana fue de 42 años, con un rango entre 33 y 53. Estos valores están en concordancia con los de todos los estudios previos que se han realizado.

Los efectos adversos descritos más frecuentes del tratamiento con acupuntura son hematomas, mareos, náuseas y aumento del dolor en la zona puncionada ${ }^{56,57}$. En el presente estudio ningún paciente refirió efectos adversos o intolerancia al tratamiento. De los estudios previos ${ }^{40-52}$, ninguno informa sobre los efectos adversos de los tratamientos.

El dolor medido en la EVA constituyó el parámetro sobre el que se fundamentaron los diferentes criterios de valoración de respuesta del dolor a los tratamientos: respuesta clínica relevante, distribución temporal del efecto del tratamiento y estabilidad del efecto del tratamiento.

De entre estos criterios, consideramos como variable principal al porcentaje de pacientes que alcanzaron respuesta clínica relevante (reducción del dolor mayor del $50 \%$ del basal o dolor menor de 30 en la EVA) tal y como hicieron Katsoulis y cols. ${ }^{38}$ en su estudio, ya que refleja con mayor rigor la eficacia de los tratamientos frente a la diferencia en los valores EVA pre y postratamiento.

Con respecto a la distribución temporal del efecto del tratamiento se observó que el tratamiento de acupuntura tiene un efecto rápido de disminución del dolor y que a partir de los tres meses prácticamente no hay respuestas clínicas relevantes. Esta distribución temporal de las respuestas clínicas relevantes no es debida al azar.

Con respecto a la disminución cuantitativa del dolor en la EVA, se observó a lo largo del seguimiento una reducción de la intensidad del dolor. El descenso de la puntuación EVA (intragrupo) fue estadísticamente significativo.

Con respecto a la estabilidad del efecto del tratamiento, nos interesó especialmente el análisis de dicha estabilidad, dado que el tratamiento fue aplicado solamente durante cuatro semanas al principio del estudio. No existieron diferencias significativas en la puntuación EVA a partir del tercer mes, manteniéndose estable el efecto del tratamiento y la mejoría inicial.

Con respecto a la MAO, nuestro estudio incluyó pacientes con SDM de la MM en los cuales no hubo ninguna sintomatología articular. Esto explica que los valores basales de MAO resultaron normales (mayores a $35 \mathrm{~mm}$ ). Como se aprecia comparando la apertura final con la apertura inicial, hubo un ligero aumento estadísticamente significativo, que se mantiene estable a lo largo del seguimiento.

Del estudio de Smith y cols. ${ }^{2}$ tomamos la variable "Perspectiva funcional del paciente o grado de disfunción percibida en la EVA" que consideramos un parámetro más adecuado que los cuestionarios de calidad de vida, para evaluar la repercusión del SDM de MM; dado que no existe ninguno diseñado específicamente para el SDM de la $\mathrm{MM}^{58,59}$.

En nuestro estudio, hubo una mejoría del grado de disfunción percibida a lo largo del seguimiento, de manera que la disfunción percibida final presentó diferencias estadísticamente significativas con respecto a la inicial.

En el estudio de Smith y cols. ${ }^{2}$ los valores basales del grado de disfunción percibida en la EVA fueron de 52,76 en el grupo de acupuntura real y de 13,33 en el grupo de acupuntura placebo. Los valores finales fueron de 23,2 y 12,5 respectivamente.
La mejoría del grado de disfunción percibida fue significativa solo en el grupo de acupuntura real. Hay que destacar la gran diferencia en los valores basales entre los dos grupos, aunque los autores no especificaron si esta diferencia fue significativa y si los grupos fueron homogéneos para esta variable; lo cual obliga a interpretar con cautela el análisis de dicha variable en este estudio.

Con respecto a los estudios similares realizados previamente, las aportaciones que realiza nuestro estudio son la selección de pacientes con dolor miofascial de la musculatura masticatoria, sin componente clínico articular y el seguimiento de un año.

Son necesarios más estudios para establecer una validación de los puntos o combinaciones de puntos de acupuntura para el tratamiento del SDM de la MM y esquemas de tratamiento homogéneos para los estudios de este tipo; a pesar de que el tratamiento con acupuntura tiene una característica fundamental que es la individualización del tratamiento.

\section{Conclusiones}

1. Los resultados del estudio demuestran que la acupuntura es eficaz en el control del dolor del SDM de la MM.

2. Los efectos terapéuticos de la acupuntura son de inicio temprano ( $<3$ meses), estables y se mantienen hasta el final del seguimiento de un año.

3. La acupuntura es un tratamiento seguro, con una incidencia de eventos adversos muy baja.

\section{Conflicto de intereses y financiación}

Los autores declaran no tener ningún conflicto de intereses.

Este estudio ha sido realizado con la ayuda del Fondo de Investigaciones Sanitarias (FIS) concedida por el Instituto de Salud Carlos III, Proyecto de Investigación 10/02857 y la Beca de Investigación "Dr. Gómez Iglesias" otorgado por la Sociedad Española de Cirugía Oral y Maxilofacial (SECOM).

\section{B I B L I O G R A F Í A}

1. Martín-Granizo R, de Pedro Marina M, Salmerón JI, Sastre J. Manual de Cirugía Oral y Maxilofacial. 2 a ed. Madrid; 2004.

2. Smith P, Mosscrop D, Davies S, Sloan P, Al-Ani Z. The efficacy of acupuncture in the treatment of temporomandibular joint myofascial pain: A randomised controlled trial. J Dent 2007;35(3):259-67. DOI: 10.1016/j.jdent.2006.09.004.

3. Lipton JA, Ship JA, Larach-Robinson D. Estimated prevalence and distribution of reported orofacial pain in the United States. J American Dent Assoc 1993;124(10):115-21.

4. List T, Wahlind $\mathrm{K}$, Wenneberg B, Dworkin SF. TMD in children and adolescents: prevalence of pain, gender differences, and perceived treatment need. J Orofac Pain 1999;13(1):9-20.

5. Manfredini D, Piccotti F, Ferronato G, Guarda-Nardini L. Age peaks of different RDC/TMD diagnoses in a patient population. J Dent 2010;38(5):392-9. DOI: 10.1016/j.jdent.2010.01.006.

6. Simons DG, Travell J. Myofascial trigger points, a possible explanations. Pain 1981;10(1):106-9.

7. Filshie J, White A. Medical acupuncture: a western scientific approach. Edinbugh: Churchill Livingstone; 1997. 
8. Vas J, Perea-Milla E, Mendez C, Silva LC, Herrera A, Aranda JM, et al. Efficacy and safety of acupuncture for the treatment of non-specific acute low back pain: a randomized controlled multicentre trial protocol. BMC Complement Alternative Med 2006;6:14. DOI: 10.1186/1472-6882-6-14

9. Vas J, Perea-Milla E, Mendez C, Herrera A, Madrazo F, Medina I, et al. Acupuncture and rehabilitation of the painful shoulder: study protocol of an ongoing multicentre randomized controlled clinical trial. BMC Complement Alternative Med 2005;5:19. DOI: 10.1186/1472-6882-5-19.

10. Kung YY, Chen FP, Chaung HL, Chou CT, Tsai YY, Hwang SJ. Evaluation of acupuncture effect to chronic myofascial pain syndrome in the cervical and upper back regions by the concept of Meridians. Acupunct Electrother Res 2001;26(3):195-202.

11. Cummings TM, White AR. Needling therapies in the management of myofascial trigger point pain: a systematic review. Arch Phys Med Rehabil 2001;82(7):986-92. DOI: 10.1053/ apmr.2001.24023.

12. Birch S, Jamison RN. Controlled trial of Japanese acupuncture for chronic myofascial neck pain: assessment of specific and non-specific effects of treatment. Clin J Pain 1998;14(3):248-55.

13. Lundeberg T, Hurting T, Lundberg S, Thomas M. Long-term results of acupuncture in chronic head and neck pain. Pain Clinic 1988;2(1):15-31.

14. Millstein-Prentky S, Olson RE. Predictability of treatment outcome in patients with myofascial pain-dysfunction (MPD) syndrome. J Dent Res 1979;58(4):1341-6.

15. Edwards J, Knowles N. Superficial dry needling and active stretching in the treatment of myofascial pain. Acupunct Med 2003;21(3):80-6.

16. Ga H, Choi JH, Park CH, Yoon, HJ. Acupuncture needling versus lidocaine injection of trigger points in myofascial pain syndrome in elderly patients - a randomized trial. Acupunct Med 2007;25(4):130-36.

17. Ga H, Koh HJ, Choi JH, Kim CH. Intramuscular and nerve root stimulation vs lidocaine injection of trigger points in myofascial pain syndrome. J Rehabil Med 2007;39(5):374-78. DOI: 10.2340/16501977-0058.

18. Ga H, Choi JH, Park CH, Yoon, HJ. Dry needling of trigger points with and without paraspinal needling in myofascial pain syndromes in elderly patients. Journal Alternative Complement Med 2007;13(6):617-24. DOI: 10.1089/acm.2006.6371.

19. Criscuolo CM. Interventional approaches to the management of myofascial pain syndrome. Curr Pain Headache Rep 2001;5(5):407-11.

20. Borg-Stein J, Simons DG. Focused review: myofascial pain. Arch Phys Med Rehabil 2002;83(3):40-9.

21. Biedermann HJ, Lapeer GL, Mauri M, McGhie A. Acupuncture and myofascial pain: treatment failure after administration of tricyclic antidepressants. Med Hypotheses 1986;19(4):397-402.

22. Gerwin RD. A review of myofascial pain and fibromyalgia - factors that promote their persistence. Acupunct Med 2005;23(3):121-34.

23. Ilbuldu E, Cakmak A, Disci R, Aydin R. Comparison of laser, dry needling, and placebo laser treatments in myofascial pain syndrome. Photomed Laser Surg 2004;22(4):306-11.

24. Wang C, Long X, Zhu X. A study on the clinical curative effect by acupuncture for myofascial pain dusfunction syndrome. Zhonghua Kou Qiang Yi Xue Za Zhi 1998;33(5):273-5.

25. Terayama H, Yamakazi H, Kanazawa T, Suyama K, Tanaka O, Sawada M, et al. Multi-acupuncture point injections and their anatomical study in relation to neck and shoulder pain syndrome (so-called Katatori) in Japan. PLoS ONE 10(6):E-0129006. DOI: 10. 1371/journal.pone.0129006.

26. Aranha MFM, Müller CEE, Gaviao MBD. Pain intensity and cervical range of motion in women with myofascial pain treated with acupuncture and electroacupuncture: a double-blinded, randomized clinical trial. Braz J Phys Ther 2015;19(1):34-43. DOI: 10.1590/bjpt-rbf.2014.0066.
27. Chow L-W, Hsieh Y-L, Kuan T-S, Hong C-Z. Needling therapy for myofascial pain: recommended technique with multiple rapid needle insertion. BioMedicine 2014;4(2):39-46. DOI: 10.7603/s40681-014-0013-2.

28. Ma Y, Bu H, Jia JR, Liu Z. Myofascial pain syndrome treated with sparrow-pecking moxibustion at trigger points: a randomized controlled trial. Zhongguo Zhen Jiu 2014;34(11):1073-5.

29. Zhao H. Clinical observation on therapeutic effect of cupping combined with acupuncture stimulation at trigger points for lumbar myofascial pain syndrome. Zhongguo Zhen Ci Yan Jiu 2014;39(4):324-8.

30. Chen RX, Kang MF, He WL, Chen SY, Zhang B. Moxibustion on heat-sensitive acupoints for treatment of myofascial pain syndrome: a multi-central randomized controlled trial. Zhongguo Zhen Jiu 2008;28(6):395-8.

31. Ma Y, Bu H, Jia JR, Zhang X. Progress of research on acupuncture at trigger point for myofascial pain syndrome. Zhongguo Zhen Jiu 2012;32(6):573-6.

32. Chou LW, Hsieh YL, Chen HS, Hong CZ, KAO mj, Han TI. Remote therapeutic effectiveness of acupuncture in treating myofascial trigger point of the upper trapezius muscle. Am J Phys Med Rehabil 2011;90(12):1036-49. DOI: 10.1097/ PHM.0b013e3182328875.

33. Wu F, Kang MF, Xiong P, Xiong J. Clinical randomized controlled trials of treatment of neck-back myofascial pain syndrome by acupuncture of Ashipoints combined with moxibustion of heat-sensitive points. Zhen Ci Yan Jiu 2011;36(2):116-20.

34. Biella G, Sotgiu ML, Pellegata G, Paulesu E, Castiglioni I, Fazio F. Acupuncture produces central activations in pain regions. Neuroimage 2001;14:60-6. DOI: 10.1006/nimg.2001.0798.

35. Han JS. Neurochemical basis of acupuncture. Annu Rev Pharmacol Toxicol 1982;22:193-220. DOI: 10.1146/annurev. pa.22.040182.001205.

36. Wu MT, Hsieh JC, Yang CF, Pan HB, Chen YC, Tsai G, et al. Central nervous pathway for acupuncture stimulation: localization of processing with functional MR imaging of the brainpreliminary experience. Neuroradiology 1999;212(1):133-41.

37. Yang C. A neuromagnetic study of acupuncturing Li4 (Hegu). Acupuncture Elect Res 1995;20(1):15-20.

38. Katsoulis J, Ausfeld-Hafter B, Windecker-Getaz I, Katsoulis K, Blagojevic N, Mericske-Stern R. Laser acupuncture for myofascial pain of the masticatory muscles. A controlled pilot study. Res Science 2010;120(3):213-25.

39. Ernst E, White AR. Acupuncture as a treatment for temporomandibular joint dysfunction. A systematic review of randomized trials. Arch Otolaryngol Head Neck Surg 1999;125(3):269-72.

40. Raustia AM, Pohjola RT, Virtanen KK. Acupuncture compared with stomatognathic treatment for TMJ dysfunction. Part I: A randomised study. J Prosthet Dent 1985;54(4):581-5.

41. Raustia AM, Pohjola RT, Virtanen KK. Acupuncture compared with stomatognathic treatment for TMJ dysfunction. Part II: components of the dysfunction index. J Prosthet Dent 1986;55(3):372-76.

42. Raustia AM, Pohjola RT, Virtanen KK. Acupuncture compared with stomatognathic treatment for TMJ dysfunction. Part III: Effects of treatment on mobility. J Prosthet Dent 1986;56(5):616-23.

43. Johansson A, Wennerberg B, Wagersten C, Haraldson T. Acupuncture of facial muscular pain. Acta Odontol Scand 1991;49(3):153-8.

44. List T, Helkimo M, Andersson S, Carlsson GE. Acupuncture and occlusal splint therapy in the treatment of craniomandibular disorders Part I. A comparative study. Swed Dent J 1992;16(4):125-41.

45. List T, Helkimo M, Andersson S, Carlsson GE. Acupuncture and occlusal splint therapy in the treatment of craniomandibular disorders Part II. A 1-year follow up study. Acta Odontol Scand 1992;50(6):375-85. 
46. List T, Helkimo M, Karlsson R. Pressure pain thresholds in patiets with craniomandibular disorders before and after treatment with acupuncture and occlusal splint therapy: a controlled clinical study. J Orofacl Pain 1993;7(3):275-82.

47. Vicente-Barrero M, Yu-Lu SL, Zhang B, Bocanegra-Pérez S, Durán-Moreno D, López-Márquez A, et al. The efficacy of acupuncture and decompression splints in the treatment of temporomandibular joint pain-dysfunction syndrome. Med Oral Patol Oral Cir Bucal 2012;17(6):e1028-33.

48. Goddard G, Karibe H, Villafuerte E. Acupuncture and sham acupuncture reduce muscle pain in myofascial patients. J Orofac Pain 2002;16(1):71-6.

49. Goddard G, Shen Y, Steele B, Springer N. A controlled trial of placebo versus real acupuncture. J Pain 2005;6(4):237-42. DOI: 10.1016/j.jpain.2004.12.009.

50. Schmid-Schwap M, Simma-Kletschka I, Stockner A, Sengstbratl M, Gleditsch J, Kundi M, et al. Oral acupuncture in the therapy of craniomandibular dysfunction syndrome - a randomized controlled trial (RTC). Wien Klin Wochenschr 2006;118:36-42. DOI: 10.1007/s00508-005-0501-1.

51. Shen YF, Younger J, Goddard G, Mackey S. Randomized clinical trial of acupuncture for myofascial pain of jaw muscles. J Orofac Pain 2009;23(4):353-59.

52. Simma I, Gleditsch JM, Simma L, Piehslinger E. Immediate effects of microsystem acupuncture in patients with oromyofascial pain and craniomandibular disorders (CMD): a doubleblind, placebo-controlled trial. British Dental Journal 2009;207(12):E26. DOI: 10.1038/sj.bdj.2009.959.
53. La Touche R, Angulo-Díaz-Parreño S, de-la-Hoz JL, FernándezCarnero J, Ge HY, Linares MT, Mesa J, Sánchez-Gutiérrez J. Effectiveness of acupuncture in the treatment of temporomandibular disorders of muscular origin: a systematic review of the last decade. J Altern Complement Med 2010;16(1):107-12. DOI: $10.1089 / \mathrm{acm} .2008 .0484$.

54. Jung A, Shin B-C, Lee M-S, Sim H-S, Ernst E. Acupuncture for treating temporomandibular joint disorders: a systematic review and meta-analysis of randomized, sham-controlled trials. J Dent 2011;39(5):341-50. DOI: 10.1016/j.jdent.2011.02.006.

55. Wilkes C. Internal derangements of the temporomandibular joint pathological variations. Arch Otolaryngol Head Neck Surg 1989;115(4):469-77.

56. Norheim AJ. Adverse effects of acupuncture. Lancet 1995;345(8964):1576.

57. List T, Helkimo M. Adverse events of acupuncture and occlusal splint therapy in the treatment of craniomandibular disorders. Cranio 1992;10(4):318-26.

58. Venere D, Corsalini M, Stefanachi G, Tafuri S, Tommaso M, Cervinara F, et al. Quality of life in fibromyalgia patients with craniomandibular disorders. Open Dent J 2015;9:9-14. DOI: 10.2174/1874210601509010009.

59. Karibe H, Goddard G, Shimazu K, Kato Y, Warita-Naoi S, Kawakami T. Comparison of self-reported pain intensity, sleeping difficulty and treatment outcomes of patients with myofascial temporomandibular disorders by age group: a prospective outcome study. BMC Musculoskelet Dis 2014;15:423. DOI: 10.1186/1471-2474-15-423. 